\title{
Características Clínicas e Demográficas de 193 Pacientes com Febre Reumática
}

\section{Clinical and Demographic Features of 193 Patients with Rheumatic Fever}

\author{
Maria Teresa Ramos Ascensão Terreri(1), Álvaro Manuel Caldas ${ }^{(2)}$, Cláudio Arnaldo Len ${ }^{(3)}$, Fabio \\ Ultchak $^{(4)}$, Maria Odete Esteves Hilário ${ }^{(5)}$
}

\section{RESUMO}

Objetivo: avaliar as características clínicas e demográficas de pacientes com febre reumática aguda (FRA), acompanhados no período de 1995 a 2005. Métodos: foram avaliados, retrospectivamente, os prontuários de 193 pacientes com FRA diagnosticada de acordo com os critérios de Jones revisados (1992). Foram incluídos apenas os pacientes que iniciaram o acompanhamento nos primeiros dois meses de doença para que os erros diagnósticos fossem diminuídos. Foram analisados dados demográficos, características clínicas e laboratoriais, bem como anormalidades ecocardiográficas dos pacientes. Resultados: 4 dos $193(2,1 \%)$ pacientes eram menores de 5 anos de idade. Dentre as manifestações clínicas, a artrite foi a mais freqüente $(70,5 \%)$ seguida da cardite $(50,8 \%)$ e coréia $(35,2 \%)$. Em 64 pacientes $(33,2 \%)$, a artrite foi atípica, ou seja, foi mon oarticular $(10,9 \%)$ ou comprometeu articulações não-usuais $(59,4 \%)$, ou teve duração maior do que seis semanas ( $18,8 \%$ ), ou não respondeu ao ácido acetilsalićlico (10,9\%). Em relação ao comprometimento cardíaco, observamos cardite subclínica em 19\% dos pacientes. A cardite clínica teve como alteração valvar mais encontrada a regurgitação mitral (96,9\% dos pacientes com cardite). A coréia esteve presente em 35\% dos casos. Quanto ao laboratório, anemia $(\mathrm{p}=0,01)$, VHS (velocidade de hemossedimentação) $\geq 100 \mathrm{~mm}(\mathrm{p}=0,04) \mathrm{e}$ elevação de $\alpha \mathrm{l}$ glicoproteína ácida ( $\mathrm{p}=0,04)$ foram estatisticamente mais freqüentes nos pacientes com cardite do que naqueles sem este comprometimento. Em 15\% dos pacientes houve recorrências. Conclusão: 1) a febre reumática ainda é prevalente em nosso meio; 2) a presença da artrite atípica é um achado freqüente, devendo ser devidamente valorizada para o diagnóstico da FRA; 3 ) a cardite subclínica pode ocorrer e deve ser considerada; 4 ) a nossa

\begin{abstract}
Objective: the aim of this study was to analyze the demographic and clinical characteristics of patients with acute rheumatic fever (ARF), followed between 1995 and 2005. Methods: we retrospectively reviewed the medical records of 193 patients with ARF diagnosed according to the revised Jones criteria (1992). Only the patients that initiated the follow-up in the first two months of onset were included, in order to reduce the diagnostic mistake. Demographic, clinical and laboratorial data and echocardiographic abnormalities were considered. Results: four out of $193(2.1 \%)$ were younger than 5 years old. The most frequent clinical manifestation was arthritis (70.5\%) followed by carditis (50.8\%) and chorea (35.2\%). Atypical arthritis occurred in $64(33.2 \%)$ patients characterized by monoarthritis (10.9\%), or involvement of unusual joints (59.4\%), or duration longer than 6 weeks (18.8\%), or poor response to salicylates (10.9\%). Regarding the cardiac involvement we observed subclinical carditis in 19\% of the patients. Clinical carditis presented most frequently as mitral involvement (regurgitation) (96.9\%). Chorea was present in $35 \%$ of the patients. Regarding lab work, anemia $(p=0.01)$, erythrocyte sedimentation rate $\geq 100 \mathrm{~mm}(p=0.04)$ and elevation of $\alpha 1$ acid glycoprotein $(p=0.04)$ were statistically more frequent in patients with carditis compared to patients without this involvement. Fifteen percent of patients experienced recurrences. Conclusion: 1) rheumatic fever is still prevalent in our environment; 2) atypical arthritis is a common finding and must be taken into account in the ARF diagnosis; 3) subclinical carditis must be considered in our patients; 4) the frequency of
\end{abstract}

\footnotetext{
Disciplina de Alergia, Imunologia Clínica e Reumatologia do Departamento de Pediatria da Universidade Federal de São Paulo (UNIFESP). Recebido em 02/09/06. Aprovado, após revisão, em 02/11/06.

1. Professora Afiliada da Disciplina de Alergia, Imunologia Clínica e Reumatologia do Departamento de Pediatria da UNIFESP

2. Mestre em Reumatologia Pediátrica

3. Professor Adjunto da Disciplina de Alergia, Imunologia Clínica e Reumatologia do Departamento de Pediatria da UNIFESP

4. Pediatra da UNIFESP.

5. Professora Livre Docente, Chefe do Setor de Reumatologia da Disciplina de Alergia, Imunologia Clínica e Reumatologia do Departamento de Pediatria da UNIFESP.

Endereço para correspondência: Maria Teresa Terreri, Rua Loefgreen, 2381, apto.141, CEP 04040-004, São Paulo, SP, Brasil, telefone/fax: (11) 5579-1590, e-mail: terreri@uninet.com.br
} 
freqüência de coréia de Sydenham continua superior à descrita na literatura; 5 ) recorrências são freqüentes em nossos pacientes.

Palavras-chave: febre reumática, cardite subclínica, artrite atípica, coréia.

\section{INTRODUÇÃO}

A febre reumática (FR) é uma complicação tardia, inflamatória, não supurativa da infecção das vias aéreas superiores pelo estreptococo beta hemolítico do grupo A de Lancefield (EBHA). Embora a incidência da FR tenha diminuído nos últimos 30 anos nos países desenvolvidos, ela ainda representa um problema de saúde pública com altas morbidade e mortalidade nos países em desenvolvimento ${ }^{(1)}$.

O diagnóstico da FR é baseado em uma combinação de achados clínicos e laboratoriais ${ }^{(2)}$. Entretanto, devido à grande variação de apresentações clínicas, o diagnóstico da FR constitui um desafio para o clínico ${ }^{(3)}$.

A artrite é a manifestação clínica mais comum da FR com evolução favorável em cerca de três semanas, na grande maioria dos casos. Entretanto, apresentações atípicas podem ocorrer, incluindo quadros monoarticulares, duração superior a seis semanas e pobre resposta aos salicilatos, além de acometimento de articulações como quadris, coluna cervical e pequenas articulações ${ }^{(4-6)}$. A cardite é a manifestação clínica mais grave da FR contribuindo para a doença valvar, seqüelas a longo prazo e até mesmo óbito ${ }^{(7)}$. A coréia de Sydenham é a manifestação menos encontrada, caracterizada por uma síndrome hipotônica e hipercinética com movimentos involuntários, geralmente bilaterais, de membros e face.

Por ser a FR uma doença prevalente e de grande interesse em nosso meio, cujo diagnóstico continua representando um desafio para os pediatras e reumatologistas, nos sentimos motivados a avaliar as características clínicas e demográficas dos pacientes acompanhados em nosso Serviço nos últimos dez anos com o objetivo de melhor caracterizá-los.

\section{PACIENTES E MÉTODOS}

Foram selecionados, consecutivamente, 193 pacientes com febre reumática aguda (FRA) de acordo com os critérios de Jones revisados, cujo acompanhamento em nosso Serviço foi iniciado nos primeiros dois meses da doença $a^{(2)}$.

Este estudo foi retrospectivo, baseado na revisão e análise de prontuários médicos. Foram avaliados os dados demográficos, as características clínicas e laboratoriais, bem como as anormalidades ecocardiográficas dos 193 pacientes.
Sydenham's chorea is higher than that described in the literature; 5) recurrences are frequent in our patients.

Keywords: rheumatic fever, subclinical carditis, atypical arthritis, chorea.

Foi definida como artrite típica aquela que acometia duas ou mais articulações, grandes articulações, com duração menor que seis semanas e resposta satisfatória ao antiinflamatório não-hormonal (AINH). A cardite clínica foi considerada na presença de sopro audível e alteração correspondente ao ecocardiograma. A presença de achados ecocardiográficos de lesões valvares orgânicas, na ausência de manifestação clínica de cardite (sopro cardíaco) foi definida como cardite subclínica. Para melhor caracterizar a cardite subclínica, avaliamos, prospectivamente e de maneira cega, 56 dos 193 pacientes da amostra geral e 20 controles (crianças saudáveis), através do exame clínico (pelo cardiologista), do ecocardiograma Doppler colorido, do eletrocardiograma e da radiografia de tórax, na fase aguda e com três e seis meses, e dois e cinco anos de evolução.

Os parâmetros laboratoriais de anormalidade adotados foram hemoglobina $<11 \mathrm{mg} / \mathrm{dl}$, velocidade de hemossedimentação (VHS) $\geq 25 \mathrm{~mm}$ na primeira hora, proteína C-reativa $($ PCR $)>6,5 \mathrm{mg} / \mathrm{dl}$, antiestreptolisina $\mathrm{O}($ ASLO $)>250$ UI, gamaglobulina $\geq 1,4 \mathrm{~g} / \mathrm{dl}$ e $\alpha \mathrm{l}$ glicoproteína ácida $\geq$ $140 \mathrm{mg} / \mathrm{dl}$.

A recorrência foi considerada quando houve aparecimento de novo surto após um período sem atividade clínica e laboratorial de pelo menos 60 dias.

$\mathrm{Na}$ análise estatística, foi utilizado o teste de qui-quadrado e foi considerado significante, $\mathrm{p}<0,05$.

\section{RESULTADOS}

Dentre os 193 pacientes, $111(57,5 \%)$ eram do sexo feminino e $100(51,8 \%)$ não-caucasóides, com média de idade na ocasião do surto de 9,9 anos (variação de 3 a 15 anos). Quatro dos $193(2,1 \%)$ eram menores de cinco anos de idade.

A média do intervalo entre as primeiras manifestações e o diagnóstico foi de 27 dias, com variação de zero a 60 dias.

Em relação ao número de surtos, 164 (85\%) pacientes apresentaram apenas um surto, 23 pacientes $(11,9 \%)$ dois, cinco pacientes $(2,6 \%)$ três e um paciente $(0,5 \%)$ quatro surtos de FR em um período médio de evolução de três anos e meio. Portanto, 29/193 (15\%) pacientes apresentaram pelo menos uma recorrência. 
O início da FR ocorreu no verão em 58 (30,9\%) pacientes, no outono em $56(29,8 \%)$, no inverno em 42 (22,3\%) e na primavera em 32 (17\%) pacientes. Em cinco pacientes não foi possível obter este dado.

O tempo de latência entre a infecção estreptocócica e o diagnóstico de FR pôde ser determinado em 84 pacientes $(43,5 \%)$. Destes, $19(22,6 \%)$ pacientes tiveram período de latência de zero a sete dias, $49(58,3 \%)$ de oito a 21 dias e $16(19 \%)$ maior que 21 dias.

Em 26/185 (14,1\%) pacientes foi relatada história familiar de FR em parentes de primeiro ou segundo grau.

Em relação às manifestações clínicas do surto agudo de FR, 136/193 (70,5\%) pacientes apresentaram artrite (50\% meninas). Quanto à cardite, ela foi diagnosticada em 98 $(50,8 \%)$ pacientes $(63 \%$ meninas $)$, tendo sido isolada em $7(7,1 \%)$. A coréia ocorreu em $68(35,2 \%)$ pacientes $(76 \%$ meninas). Em $35(51,4 \%)$ ela foi associada à cardite $\mathrm{e} / \mathrm{ou}$ artrite e em 33 (48,5\%) foi manifestação isolada. Os nódulos subcutâneos e o eritema marginado ocorreram em 2,1\% e 2,6\% dos pacientes, respectivamente.

A artrite se manifestou na forma oligoarticular $(\leq 4$ articulações acometidas) em 81/128 (63,3\%) pacientes e foi migratória em 83/114 (72,8\%). Em oito pacientes, o número de articulações acometidas não pôde ser determinado. Os tornozelos e os joelhos foram as articulações mais acometidas em 99/136 (72,8\%) e 95/136 (69,9\%) pacientes, respectivamente.

Em 64 pacientes $(33,2 \%)$, a artrite manifestou-se de forma atípica, ou seja, acometeu pequenas articulações (como metacarpofalangianas, interfalangianas proximais e interfalangianas distais), quadris ou coluna cervical em $59,4 \%$, teve duração maior de seis semanas em $18,8 \%$, foi monoarticular em $10,9 \%$ e teve resposta insatisfatória ao AINH em 10,9\% dos casos.

A cardite teve como alteração valvar mais encontrada a regurgitação mitral em 95 pacientes $(96,9 \%$ dos pacientes com cardite), tendo sido isolada em 68 e associada à regurgitação aórtica em 27 pacientes. Em apenas três a regurgitação aórtica manifestou-se isoladamente. Na Tabela 1 , estão relacionadas as valvas mais acometidas e as lesões mais encontradas nos pacientes com cardite.

Dos 56 pacientes com FRA avaliados prospectivamente, $27(48,2 \%)$ apresentaram cardite clínica na primeira avaliação. Dos 29 pacientes com artrite e/ou coréia sem a presença de cardite clínica, 11 (37,9\%) apresentaram ao ecocardiograma alterações compatíveis com lesão orgânica, que persistiram em oito $(72 \%)$ pacientes durante o período de evolução. Um dado interessante é que dos $10 / 29$ pacien-
TABELA 1

DISTRIBUIÇÃO DAS ALTERAÇÕES VALVARES ENCONTRADAS NOS PACIENTES COM CARDITE REUMÁTICA, DE ACORDO COM A VALVA ACOMETIDA, O TIPO E O GRAU DE ACOMETIMENTO $(\mathrm{N}=98)$

\begin{tabular}{l|c|c}
\hline ALTERAÇÃo VALVAR & $\mathbf{n}$ & $\%$ \\
\hline Regurgitação mitral & $95 / 98$ & 96,9 \\
\hline Leve & $58 / 95$ & 61,1 \\
\hline Moderada & $25 / 95$ & 26,3 \\
\hline Grave & $8 / 95$ & 8,4 \\
\hline Ignorada & $4 / 95$ & 4,2 \\
\hline Regurgitação aórtica & $30 / 98$ & 30,6 \\
\hline Leve & $22 / 30$ & 73,3 \\
\hline Moderada & $7 / 30$ & 23,3 \\
\hline Grave & $1 / 30$ & 3,3 \\
\hline Espessamento mitral & $30 / 98$ & 30,6 \\
\hline Mínimo & $24 / 30$ & 80,0 \\
\hline Leve & $6 / 30$ & 20,0 \\
\hline Espessamento aórtico & $6 / 98$ & 6,1 \\
\hline Mínimo & $3 / 6$ & 50,0 \\
\hline Leve & $3 / 6$ & 50,0 \\
\hline
\end{tabular}

tes deste grupo com coréia isolada, 8 apresentaram cardite subclínica. O grupo controle não apresentou alterações ao ecocardiograma.

A coréia esteve presente em 68/193 (35,2\%) pacientes com média de duração de 78,8 dias (variação de 18 a 300 dias). Ela foi a única manifestação da FR em $48 \%$ dos pacientes e se apresentou na forma generalizada em 49/66 $(74,2 \%)$ e como hemicoréia em $17 / 66(25,8 \%)$ pacientes. Em dois pacientes não foi possível obter este dado.

Alterações da escrita e da fala foram observadas em $49 / 68(72,1 \%)$ pacientes, fraqueza muscular em 48/68 (70,6\%) e distúrbios da marcha em 46/68 (67,6\%). Instabilidade emocional ocorreu em 41/62 (66,1\%) pacientes, irritabilidade em sete, choro fácil em dois, agitação em um e cansaço em um.

Na Tabela 2, estão relacionadas as alterações laboratoriais dos pacientes com e sem cardite clínica. Observamos que os pacientes com cardite apresentaram mais freqüentemente anemia $(p=0,01)$, elevação de VHS acima de $100 \mathrm{~mm} / \mathrm{l} \stackrel{\mathrm{a}}{\mathrm{h}}$ hora $(\mathrm{p}=0,04)$ e de $\alpha \mathrm{l}$ glicoproteína ácida $(\mathrm{p}=0,04)$. Dos 37 pacientes que apresentaram VHS $\geq 100$ 
TABELA 2

Alterações laboratoriais dos PaCientes COM Febre REUMÁTICA, COM E SEM CARDITE $(\mathrm{N}=193)$

\begin{tabular}{|c|c|c|c|c|c|}
\hline \multirow[t]{2}{*}{ Exames laboratoriais } & \multicolumn{2}{|c|}{$\begin{array}{c}\text { Com cardite } \\
(n=98)\end{array}$} & \multicolumn{2}{|c|}{$\begin{array}{l}\text { Sem cardite } \\
\quad(n=95)\end{array}$} & \multirow[t]{2}{*}{ p } \\
\hline & $\mathrm{n}$ & $\%$ & $\mathrm{n}$ & $\%$ & \\
\hline Hemoglobina $<11 \mathrm{mg} / \mathrm{dl}$ & $37 / 95$ & 38,9 & $20 / 90$ & 22,2 & $0,01^{*}$ \\
\hline VHS $\geq 25 \mathrm{~mm} / 1^{\mathrm{a}}$ hora & $77 / 91$ & 84,6 & $52 / 93$ & 55,9 & $0,0001^{*}$ \\
\hline $\mathrm{VHS} \geq 100 \mathrm{~mm} / 1^{\mathrm{a}}$ hora & $24 / 91$ & 26,4 & $13 / 93$ & 14,0 & $0,04^{*}$ \\
\hline $\mathrm{PCR} \geq 6,5 \mathrm{mg} / \mathrm{dl}$ & $20 / 77$ & 26,0 & $19 / 72$ & 26,4 & 0,95 \\
\hline$\alpha 1$ glicoproteína ácida $\geq 140 \mathrm{mg} / \mathrm{dl}$ & $40 / 73$ & 54,8 & $25 / 69$ & 36,2 & $0,04^{*}$ \\
\hline Gamaglobulina $\geq 1,4 \mathrm{~g} / \mathrm{dl}$ & $44 / 64$ & 68,7 & $31 / 51$ & 60,8 & 0,43 \\
\hline
\end{tabular}

VHS = velocidade de hemossedimentação.

$\mathrm{PCR}=$ proteína $\mathrm{C}$ reativa.

$\mathrm{mm} / \mathrm{l}^{\mathrm{a}}$ hora, $24(64,9 \%)$ tinham cardite. Todos os pacientes com cardite isolada $(\mathrm{N}=7,3,6 \%)$ apresentaram VHS $\geq$ $100 \mathrm{~mm} / \mathrm{l}^{\underline{a}}$ hora.

Em relação à ASLO, ela se mostrou alterada na primeira dosagem em 111/163 (68,1\%) pacientes. Em uma segunda dosagem realizada em 136 pacientes, $20(14,7 \%)$ apresentaram pelo menos o dobro do título anterior e 21 $(15,4 \%)$ aumentaram o título, porém sem atingir o dobro do valor anterior.

\section{DISCUSSÃO}

Neste grupo de 193 pacientes foram incluídos somente aqueles cujo diagnóstico foi realizado em nosso Serviço nos primeiros dois meses da doença, pela necessidade de se avaliar pacientes com FR durante o surto agudo da doença, possibilitando assim a documentação dos achados clínicos e laboratoriais e a certeza do diagnóstico.

Com relação às características dos indivíduos deste estudo, observamos predomínio do sexo feminino nos pacientes com cardite e coréia o que está de acordo com dados da literatura ${ }^{(3,8)}$.

Embora a FRA seja mais freqüentemente observada em crianças e adolescentes na faixa etária dos cinco aos 15 anos, ela pode ser diagnosticada em crianças com menos de cinco anos como ocorreu com quatro dos nossos $193(2,1 \%)$ pacientes, semelhante ao encontrado em dois estudos recentes $^{(9,10)}$.

A alta taxa de recorrência encontrada se deve provavelmente à falta de profilaxia adequada. A dificuldade de acesso por parte das famílias aos locais de aplicação da penicilina benzatina e a não disponibilidade da medicação em postos de saúde, são, provavelmente, as principais razões para tal.
Quanto à distribuição em relação às estações do ano, observamos maior freqüência do diagnóstico de FRA nos meses do verão e do outono, ao contrário do que descreve a literatura (primavera e inverno) $)^{(11)}$. Tal achado talvez tenha relação com a ausência de estações do ano bem definidas em nossa região.

Embora o período de latência entre a infecção estreptocócica e as primeiras manifestações da doença tenha sido de uma a três semanas na maioria dos pacientes cujo dado pode ser obtido, ele também variou de zero a sete dias em $22,6 \%$, o que não afasta o diagnóstico de FRA.

Em relação às manifestações clínicas da FRA, a artrite foi a mais freqüente, seguida pela cardite e coréia, o que está de acordo com a literatura. Entretanto, ao contrário do que é observado em outros países, a frequiência de coréia em nosso meio é bastante elevada, como já havíamos descrito anteriormente $^{(12,13)}$. Dados da literatura descrevem uma freqüência de coréia de Sydenham em torno de 15 a 20\%(14). É possível que a maior freqüência desta manifestação em nossos pacientes esteja relacionada com características patogênicas do estreptococo.

Como pudemos observar, em uma proporção significativa dos nossos pacientes, o padrão do comprometimento articular diferiu do descrito na literatura, caracterizando a artrite atípica. Esta tem sido relatada como uma manifestação relativamente freqüente em algumas populações com alta incidência de FRA devendo ser considerada de forma cuidadosa no diagnóstico ${ }^{(4,5,6)}$. Alguns trabalhos da literatura descrevem uma entidade denominada de artrite reativa pós-estreptocócica caracterizada por artrite mono ou oligoarticular, simétrica, não-migratória, de duração prolongada, não-responsiva ao AINH e em alguns pacientes com comprometimento axial, e de aparecimento após período de latência de curta duração ${ }^{(15,16)}$. Entretanto, este quadro é considerado por nós e por outros autores como febre reumática de apresentação atípica e os pacientes devem ser tratados como tal ${ }^{(4,17)}$. Alguns dados do presente estudo e de estudos de outros autores realizados em nosso meio fundamentam este conceito $^{(4)}$.

Quanto ao comprometimento cardíaco, sua freqüência e características estão de acordo com as descritas na literatu$\mathrm{ra}^{(13,18)}$. Em relação à cardite subclínica, observada por nós em $37 \%$ dos nossos 56 pacientes avaliados, é ainda assunto controverso na literatura. Outros autores já descreveram esta apresentação ${ }^{(19,20,21,22)}$. Embora ela não faça parte dos critérios de Jones, deve ser considerada, pois as lesões valvares podem persistir na evolução em aproximadamente $70 \%$ dos pacientes ${ }^{(23)}$. A importância deste achado se relaciona 
não somente à necessidade de tratamento com corticóide como também à duração da profilaxia secundária.

A coréia de Sydenham se apresentou como manifestação isolada em menos da metade dos nossos pacientes, semelhante ao encontrado em outros trabalhos mais recentes ${ }^{(14,24)}$. Portanto, a coréia não deve mais ser considerada de apresentação predominantemente isolada como os trabalhos anteriores referiam ${ }^{(25)}$. O eritema marginado e os nódulos subcutâneos ocorreram em menos de 3\% dos nossos pacientes, o que reforça a opinião de alguns autores de que estas manifestações por serem achados tão pouco freqüentes não deveriam ter o mesmo valor dos outros critérios maiores de Jones ${ }^{(26)}$.

Quanto ao laboratório, anemia, elevação da VHS e da $\alpha 1$ glicoproteína ácida foram mais freqüentes no grupo com cardite, refletindo o processo inflamatório mais intenso. Estes são dados interessantes e ainda não descritos por outros autores.

A gamaglobulina mostrou-se elevada na maioria dos

\section{REFERÊNCIAS}

1. Gibofsk A, Kerwar S, Zabriskie JB: Rheumatic fever. The relationships between host, microbe and genetics. Rheum Dis Clin North Am 24: 237-59, 1998.

2. Dajani AS, Ayoub E, Bierman FZ: Special writing group of the committee on rheumatic fever, endocarditis and Kawasaki disease of the council on cardiovascular disease in the young of the American Heart Association: Guidelines for the diagnosis of rheumatic fever - Jones Criteria, 1992 Update. JAMA 268 : 2069-73, 1992.

3. Hilário MO, Terreri MT: Rheumatic fever and post-streptococcal arthritis. Best Pract Res Clin Rheumatol 16: 481-94, 2002.

4. Pilleggi GCS, Ferriani VPL: Manifestações articulares atípicas em crianças com febre reumática. ("Atypical articular involvement in children with rheumatic fever"). J Pediatr (Rio J) 76: 49-54, 2000.

5. Hilário MOE, Len C, Goldenberg J, Fonseca AS, Ferraz MB, Naspitz CK: Febre reumática: manifestações articulares atípicas. ("Rheumatic fever: atypical articular involvement"). Rev Assoc Med Bras 38: 214-6,1992.

6. Carapetis JR, Currie BJ: Rheumatic fever in a high incidence population: the importance of monoarthritis and low grade fever. Arch Dis Child 85: 223-7, 2001.

7. Gururaj AK, Choo KE, Ariffin WA, Sharifah A: A clinical, laboratory and echocardiographic profile of children with acute rheumatic fever. Singapore Med J 31: 364-7, 1990.

8. Majeed HA, Batnager S, Yousof AM, Khuffash F, Yusuf AR: Acute rheumatic fever and the evolution of rheumatic heart disease: a prospective 12 year follow-up report. J Clin Epidemiol 45: 871-5, 1992.

9. Tani LY, Veasy LG, Minich LL, Sahddy RE: Rheumatic fever in children younger than 5 years: is the presentation different? Pediatrics 112: 1065-8, 2003.

10. Chockalingam A, Prabhakar D, Dorairajan S, et al: Rheumatic pacientes com e sem cardite, sem diferença estatística entre os grupos. A participação da resposta imune humoral na patogênese da doença pode explicar a elevação da gamaglobulina.

Quanto à ASLO, não foi detectada elevação em seus níveis em aproximadamente $30 \%$ dos nossos pacientes mostrando a grande dificuldade que enfrentam os serviços que só dispõem deste anticorpo para o diagnóstico da infecção estreptocócica $^{(27)}$.

Em resumo, a FR continua sendo uma doença prevalente em nosso meio, com características que diferem em alguns aspectos dos conceitos estabelecidos tanto para a artrite como para a cardite. O conhecimento sobre estas diferentes formas de apresentação da doença poderá facilitar o diagnóstico e evitar os erros que ainda são freqüentes.

Declaramos a inexistência de conflitos de interesse.

heart disease occurrence, patterns and clinical correlates in children aged less than five years. J Heart Valve Dis 13: 11-4, 2004.

11. Soto Lopez ME, Cordera Gonzalez CF, Estrada L, Guel L, Abud Mendonza C, Reyes PA: Rheumatic fever in the 5-year period of 1994-1999 at 2 hospitals in San Luis Potosi and Mexico D.F. Arch Cardiol Mex 71: 127-35, 2001.

12. Goldenberg J, Ferraz MB, Hilário MO, Fonseca AS, Bastos W, Sachetti S: Increase in incidence of Sydenham's chorea in São Paulo, Brazil. J Trop Pediatr 39: 192-3, 1993.

13. Da Silva $\mathrm{CH}$ and Department of Rheumatology, Pediatrics Society of São Paulo, Brazil: Rheumatic fever: a multicenter study in the state of Sao Paulo. Pediatric Committee- São Paulo Pediatric Rheumatology Society. Rev Hosp Clin Fac Med Sao Paulo 54: 85-90, 1999.

14. Ravisha MS, Tullu MS, Kamat JR: Rheumatic Fever and Rheumatic Heart Disease: Clinical Profile of 550 cases in India. Arch Med Res 34: 382-7, 2003.

15. Ahmed S, Ayoub EM, Scornik JC, Wang CY, She JX: Poststreptococcal reactive arthritis: Clinical characteristics and association with HLA-DR alleles. Arthritis \& Rheum 41: 1096$102,1998$.

16. Birdi N, Hosking M, Clulow MK, Duffy CM, Allen U, Petty RE: Acute rheumatic fever and poststreptococcal reactive arthritis: diagnostic and treatment practices of pediatric subspecialists in Canada. J Rheumatol 28: 1681-8, 2001.

17. Gibbas DL \& Broussard DA: Poststreptococcal reactive polyarthritis - rheumatic fever or not? Arthritis \& Rheum 29 (suppl4): S92, 1986.

18. Agozzino L, Falco A, De Luca L, De Vivo F, Esposito S, Cotrufo M: Surgical pathology of the aortic valve: a morphologic study on 912 surgically excised valves. G Ital Cardiol 22: 1169-77, 1992.

19. Agarwal PK, Misra M, Sarkari NB, Gupta AK, Agarwal P: 
Usefullness of echocardiography in detection of subclinical carditis in acute rheumatic polyarthritis and rheumatic chorea. J Assoc Physicians India 46: 937-8, 1998.

20. Lanna CC, Tonelli E, Barros MV, Goulart EM, Mota CC: Subclinical rheumatic valvitis: a long-term follow-up. Cardiol Young 13: 431-8, 2003.

21. Oskutlu S, Hallioglu O, Ayabakan C: Evaluation of subclinical valvar disease in patients with rheumatic fever. Cardiol Young 13: 495-9, 2003.

22. Hilário MOE, Andrade JL, Gasparian AB, Carvalho AC, Andrade CT, Len CA: The value of echocardiography in the diagnosis and followup of rheumatic carditis in children and adolescents: a 2 year prospective study. J Rheumatol 27: 1082-6, 2000.

23. Caldas AM, Terreri MT, Moises VA, Silva CM, Carvalho AC, Hilário MOE: The case for utilizing more strict quantitative echocardiographic criterions for diagnosis of subclinical rheumatic carditis. Cardiol Young, 2006 (in press).

24. Özer S, Hallioglu O, Özkutlu S, Çeliker A: Childhood acute rheumatic fever in Ankara, Turkey. Turkish J Pediatr 47: 120-4, 2005

25. Mercadante MT, Busatto GF, Lombroso PJ, et al: The psychiatric symptoms of rheumatic fever. Am J Psychiatry 157: 2036-8, 2000 .

26. Terreri MT, Hilário MO: Diagnóstico clínico da Febre Reumática: os critérios de Jones continuam adequados? Rev Soc Bras Cardiol Est São Paulo 15: 28-33, 2005.

27. Nair PM, Philip E, Bahuleyan CG, Thomas M, Shanmugham JS, Suguna Bai NS: The first attack of acute rheumatic fever in childhood clinical and laboratory profile. Indian Pediatr 27: 241-6, 1990. 